\title{
A Hybrid Energy Harvesting Framework for Energy Efficiency in Wireless Sensor Networks Based Smart Grid Applications
}

\author{
Huseyin Ugur Yildiz*, Vehbi Cagri Gungor ${ }^{\dagger}$, and Bulent Tavli ${ }^{\ddagger}$ \\ *TED University, 06420, Ankara, Turkey, e-mail: hugur.yildiz@ @edu.edu.tr \\ ${ }^{\dagger}$ Abdullah Gul University, 38080, Kayseri, Turkey, e-mail: cagri.gungor@agu.edu.tr \\ ${ }_{\ddagger}^{\ddagger}$ TOBB University of Economics and Technology, 06560, Ankara, Turkey, e-mail: btavli@etu.edu.tr
}

\begin{abstract}
In smart grid applications, Wireless Sensor Networks (WSNs) which consist of battery limited sensor nodes are used on critical equipments of power distribution grids for monitoring purposes. WSN nodes have tight energy constraints hence it is important to reduce the energy consumption of sensor nodes due to harsh propagation characteristics of smart grid environment. One possible way to reduce the energy consumption is to utilize transmission power control where transmission powers are adjusted according to channel conditions. Another technique is to employ energy harvesting schemes to provide additional power for nodes by using environmental energy sources. Solar and electromagnetic energies are two possible environmental energy sources in outdoor substation environments. Solar energy can be efficiently exploited in a sunny day. On the other hand, electromagnetic energy can be used at any time. In this work, we propose a hybrid energy harvesting model that exploits both solar and electromagnetic energies and develop a Mixed Integer Programming (MIP) method to minimize the energy dissipation of sensor nodes. By using the MIP framework, we quantify the impact of the proposed hybrid energy harvesting model as well as transmission power control on the energy saving of nodes.

Index Terms-smart grids, wireless sensor networks, energy harvesting, energy efficiency, mixed integer programming.
\end{abstract}

\section{INTRODUCTION}

Traditional power distribution grids spread over a large area that are monitored and diagnosed through wired communications which have high capital and operational expenditures [1]. In order to overcome such high expenditures, grid monitoring can be performed by using wireless communications techniques. Wireless Sensor Networks (WSNs) are proven to be a viable solution for grid monitoring applications such as power fraud, fault detection, outage detection, power line automation, etc. The capital and operational expenditures of WSNs are cheaper than conventional wired communication techniques [2]. The integration of modern communication methods (such as WSNs) as well as monitoring, automation, and control abilities into power grid infrastructure to improve the reliability, productivity, efficiency, etc. creates the paradigm of smart grids (SGs) [3]. Although WSNs are proven to be a promising solution for SG systems, tight battery limitations of WSN nodes create a bottleneck for long term monitoring.

ISBN 978-3-903176-05-8 (C) 2018 IFIP
WSN based SG systems experience extremely harsh environmental challenges such as fading and interference caused by the generated electromagnetic fields [4]. There are several ways to combat the extreme propagation characteristics of SGs. One possible way is to employ transmission control approaches. Increasing the transmission power levels globally in the network helps to reduce the packet errors. However, the price paid for this case is the increment in total energy dissipation of the network. Another way is to adjust the transmission power level on each link according to the channel conditions (i.e., link-level) which can, potentially, prevent the excessive energy dissipation in the network.

Although transmission power control approaches may reduce the overall energy consumption in the network, additional energy can be provided to nodes by using energy harvesting approaches. In the literature, some power sources available in the SG environment are solar and electromagnetic energies [5]. Considering an outdoor SG application, solar energy can be harvested during the day time by using solar panels (or photovoltaic cells) as long as the weather is not cloudy [6]. However, if the weather is cloudy or during the night time, then solar energy cannot be harvested efficiently. At this point electromagnetic field generated by the alternating current (AC) flowing through distribution lines can be used as a backup energy source to be exploited by using harvester devices that are either clamped or not clamped around the conductor [7]. Fluctuation of the solar energy generation rate due to the stochastic nature of weather conditions motivates us to develop a hybrid energy harvesting method by using the electromagnetic energy as an alternative energy source.

Our contributions in this work are itemized as follows:

1) We propose a hybrid energy harvesting model that exploits either solar [8], [9] or electromagnetic energies [10]-[13] depending on the time and probabilistic weather conditions.

2) We develop a Mixed Integer Programming (MIP) framework which is built on top a handshaking based linklayer model employing transmission power control approaches at link-level by using the physical layer characteristics of a well known SG environment (i.e., outdoor 
$500 \mathrm{kV}$ substation [14]). The proposed MIP framework aims to minimize the energy dissipation of the maximum energy consuming node. This is critical because the lifetime of WSNs is usually limited by the lifetime of the the highest energy consuming node.

3) We quantitatively investigate the performance of the proposed hybrid energy harvesting model in terms of energy dissipation of sensor nodes by using the MIP framework through numerical evaluations.

To the best of our knowledge, this is the first study that numerically explores the effects of decrement in energy consumption of sensor nodes when employing a hybrid energy harvesting approach by using a MIP framework that combats the harsh environmental conditions of SGs with the help of transmission power control.

\section{SYSTEM MODEL}

\section{A. Wireless Channel Model}

In this work, we choose Tmote Sky motes as our WSN node platform [15]. We use log-normal shadowing channel model to account the propagation loss which has proven to be an accurate channel model [3]. The signal-to-noise ratio (i.e., $\overline{\gamma_{i j}}(l)$ in $\left.\mathrm{dB}\right)$ at the receiving node- $j$ due to the transmission from node- $i$ with power- $l$ can be expressed as

$$
\overline{\gamma_{i j}}(l)=\underbrace{\overline{P_{t x}^{a n t}}(l)-\overline{P L_{0}}+10 n \log _{10}\left(\frac{d_{i j}}{d_{0}}\right)-\overline{X_{\sigma}}}_{\overline{P_{r x, j i}^{a n t}(l)}}-\overline{P_{n}} .
$$

In this equation, $\overline{P_{t x}^{a n t}}(l)$ is antenna transmit power when using power level- $l$ (in $\mathrm{dBm}$ ), $\overline{P_{r x, j i}^{a n t}(l)}$ is the receive power (in $\mathrm{dBm}$ ), $\overline{P L_{0}}$ is the reference path loss value (in $\mathrm{dB}$ ), $n$ is the path loss exponent, $d_{i j}$ is the link distance (in meters), $d_{0}$ is the reference distance (in meters), $\overline{X_{\sigma}}$ is a zero-mean Gaussian random variable with variance $\sigma^{2}$ denoting the shadowing random variable (in $\mathrm{dB}$ ), and $\overline{P_{n}}$ is the noise floor value which is taken as $-94 \mathrm{dBm}$ for Tmote Sky platforms [15].

We adopt two different SG propagation environments namely outdoor $500 \mathrm{kV}$ substation line-of-sight (LOS) and non-line-of-sight (NLOS). Experimentally determined lognormal channel parameters for these environments are based on the field tests conducted at Georgia Power, Atlanta, GA, USA [1]. Tmote Sky motes use Chipcon CC2420 radios and reported $\overline{P_{t x}^{a n t}}(l)$ values as well as circuitry transmission power consumption values (i.e., $P_{t x}^{c r c}(l)$ ) for this radio platform is given in Table I [15]. Tmote Sky platforms use offset quadrature phase shift keying as the modulation. After including processing gain costs (i.e., $\eta=16$ ), the successful reception probability of data packet at node- $j$ due to the transmission of node- $i$ can be calculated as [14]

$$
P_{i j}^{s, L}(l)=\left(1-Q\left(\sqrt{\eta \times \gamma_{i j}(l)}\right)\right)^{L},
$$

where $\gamma_{i j}(l)$ is the signal-to-noise ratio in ordinary form and $L$ is the data packet size in bits. Similarly, $P_{i j}^{f, L}(l)=1-P_{i j}^{s, L}(l)$ shows the failure reception probability.
TABLE I: Transmission power consumption $\left(P_{t x}^{c r c}(l)\right.$ in $\left.\mathrm{mW}\right)$ and output antenna power $\left(\overline{P_{t x}^{a n t}}(l)\right.$ in $\left.\mathrm{dBm}\right)$ for each power

\begin{tabular}{|c|c|c|c|c|c|}
\hline$l$ & $P_{t x}^{c r c}(l)$ & $\overline{P_{t x}^{a n t}}(l)$ & $l$ & $P_{t x}^{c r c}(l)$ & $\overline{P_{t x}^{a n t}}(l)$ \\
\hline 3 & 25.5 & -25 & 19 & 41.7 & -5 \\
\hline 7 & 29.7 & -15 & 23 & 45.6 & -3 \\
\hline 11 & 33.6 & -10 & 27 & 49.5 & -1 \\
\hline 15 & 37.5 & -7 & 31 & 52.2 & 0 \\
\hline
\end{tabular}
level $(l)$ for the $\mathrm{CC} 2420$ radio platform [15].

\section{B. Link-Layer Model}

1) Handshaking Model: We assume a slotted communication scheme at the link-layer where a single slot encompasses durations for $M_{P}$-byte of data packet transmission (i.e., $T_{t x}\left(M_{P}\right)$ ), $M_{A}$-byte of acknowledgement (ACK) packet transmission (i.e., $T_{t x}\left(M_{A}\right)$ ), propagation delay $\left(T_{p d}\right)$, and guard times $\left(2 \times T_{\text {grd }}\right)$ which are applied at both the beginning and end of the active slot to prevent synchronization errors [14]. We choose $M_{P}=128$ bytes, $M_{A}=20$ bytes, and data rate of Tmote Sky platforms $(R)$ as $250 \mathrm{kbps}$ [15]. Hence, the active slot time is calculated as: $T_{\text {slot }}=$ $\left[2 \times T_{g r d}+T_{t x}\left(M_{P}\right)+T_{p d}+T_{t x}\left(M_{A}\right)\right]=4.78 \mathrm{~ms}$.

During an active slot a two-way handshaking should be performed successfully for a reliable communication performance. The successful handshaking is obtained when both $M_{P}$-byte and $M_{A}$-byte of data and ACK packets are successfully received at the intended nodes. Thus, the probability of successful handshaking is calculated as $P_{i j}^{s, H S}(l, k)=$ $P_{i j}^{s, M_{P}}(l) \times P_{j i}^{s, M_{A}}(k)$ where $l$ and $k$ are the transmission power levels for data and ACK packets, respectively. Considering a stop-and-wait automatic repeat request scheme, each packet has to be retransmitted $\lambda_{i j}^{l k}=\frac{1}{P_{i j}^{s, H S}(l, k)}$ times on the average.

2) Transmitter Energy Model: Transmitter node spends $P_{t x}^{c r c}(l) \times T_{t x}\left(M_{P}\right)$ Joules of energy for transmitting $M_{P^{-}}$ byte of data packet. In the rest of the active slot time (i.e., $T_{\text {slot }}-T_{t x}\left(M_{P}\right)$ seconds), the transmitter node consumes $P_{r x}^{c r c} \times\left(T_{\text {slot }}-T_{t x}\left(M_{P}\right)\right)$ Joules of energy to stay in receive/idle mode for ACK packet reception where $P_{r x}^{c r c}=69$ $\mathrm{mW}$ is the power consumption for reception [14]. $E_{P P}=$ $12.66 \mu \mathrm{J}$ is the packet processing energy which is dissipated only once during a single slot [14]. Including the effects of retransmissions with the factor of $\lambda_{i j}^{l k}$, the total energy dissipation of the transmitter node- $i$ in a single slot is obtained as

$$
\begin{aligned}
E_{t x, i j}^{l k} & =E_{P P}+\lambda_{i j}^{l k} \times\left[P_{t x}^{c r c}(l) \times T_{t x}\left(M_{P}\right)\right. \\
& \left.+P_{r x}^{c r c} \times\left(T_{s l o t}-T_{t x}\left(M_{P}\right)\right)\right] .
\end{aligned}
$$

3) Receiver Energy Model: Receiving node- $j$ spends $P_{r x}^{c r c} \times\left[T_{s l o t}-T_{t x}\left(M_{A}\right)\right]$ Joules of energy for being in receive/idle mode to receive a data packet from the transmitting node- $i$. Upon receiving the data packet, node- $j$ consumes $P_{t x}^{c r c}(k) \times T_{t x}\left(M_{A}\right)$ Joules for ACK packet transmission by using power level- $k$. Let $\varepsilon$ denote the amount of energy dissipated for reception while considering a successful handshake. If the handshaking has failed due to the ACK 


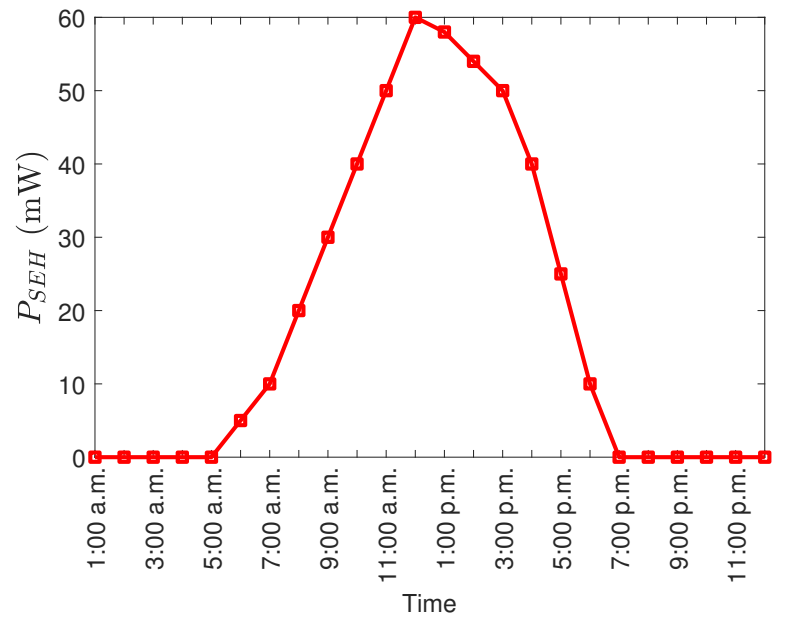

Fig. 1: Average solar harvested power $\left(P_{S E H}\right)$ of 72 days with respect to time [9].

packet errors in the reverse link, the handshaking process must be repeated where extra energy dissipation by a factor of $\frac{P_{i j}^{s, M_{P}}(l) \times P_{j i}^{f, M_{A}}(k)}{P_{i j}^{s, H S}(l, k)}$ is dissipated due to the retransmissions. Finally, if the handshaking error is caused by the failure of the data packet, then receiving node will be in receive/idle mode for the whole slot duration in which $\frac{P_{i j}^{f, M_{P}(l)}}{P_{i j}^{s, H S}(l, k)} \times\left[P_{r x}^{c r c} \times T_{\text {slot }}\right]$ Joules of energy is dissipated when considering retransmissions. Including the effects of packet processing, the total energy dissipation of the receiving node- $j$ in a single slot is calculated as

$$
\begin{aligned}
E_{r x, j i}^{l k} & =\overbrace{P_{r x}^{c r c}\left[T_{s l o t}-T_{t x}\left(M_{A}\right)\right]+\left[P_{t x}^{c r c}(k) \times T_{t x}\left(M_{A}\right)\right]}^{\varepsilon} \\
& +\frac{P_{i j}^{s, M_{P}}(l) P_{j i}^{f, M_{A}}(k)}{P_{i j}^{s, H S}(l, k)} \varepsilon+\frac{P_{i j}^{f, M_{P}}(l)}{P_{i j}^{s, H S}(l, k)}\left(P_{r x}^{c r c} \times T_{s l o t}\right) \\
& +E_{P P} .
\end{aligned}
$$

\section{Energy Harvesting Methods}

In this part, we present mechanisms for solar and electromagnetic energy harvesting methods utilized throughout this work in Sections II-C1-II-C3. Next, we describe the details of our proposed hybrid energy harvesting model in Section II-C4.

1) Solar Energy Harvester (SEH): For the solar energy harvesting (SEH) method, we assume that photovoltaic solar panels are installed on top of each WSN node. SEH profile given in [9] is adopted which is measured and averaged between the beginning of June to middle of August for 72 days [8]. In Figure 1, we present the average solar harvested power (i.e., $P_{S E H}$ ) with respect to time. As seen from this figure, solar energy can be exploited between 5:00 a.m. and 7:00 p.m. and the maximum harvested power is measured as $60 \mathrm{~mW}$ at $12: 00$ p.m.

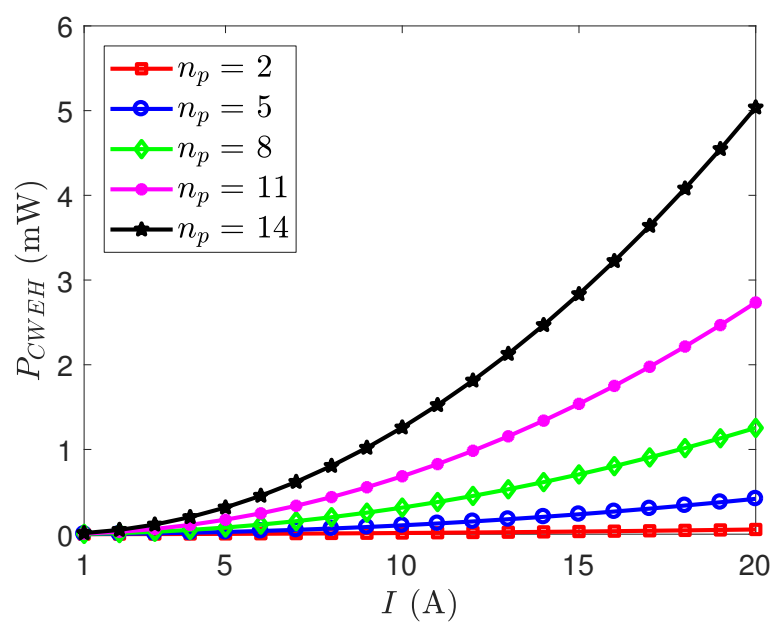

(a) Conductor winding electromagnetic harvesters (CWEH)

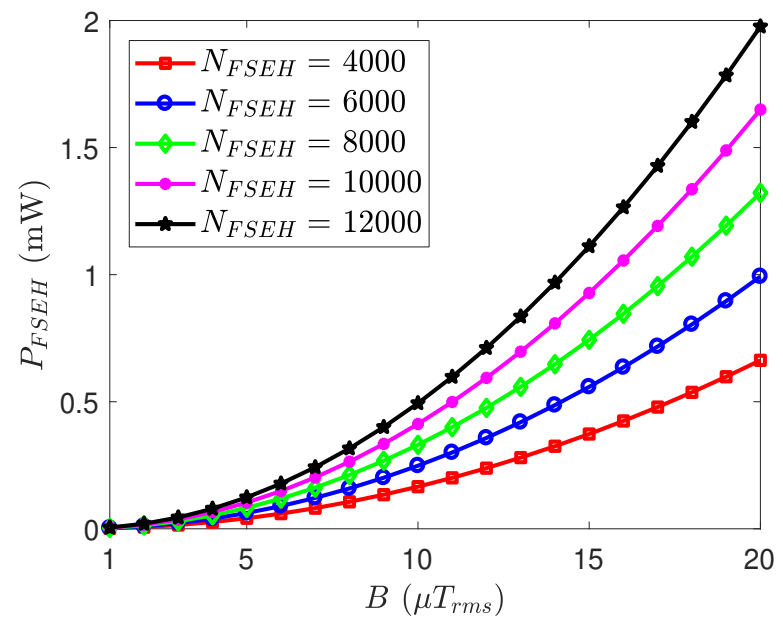

(b) Free standing electromagnetic harvesters (FSEH)

Fig. 2: Harvested powers for CWEH $\left(P_{C W E H}\right)$ and FSEH $\left(P_{F S E H}\right)$ methods.

2) Conductor Winding Electromagnetic Harvester $(C W E H)$ : A conductor winding electromagnetic harvester (CWEH) is composed of an inductive multi-turn coil where its cross sectional area is surrounding the magnetic core material which is constructed either by using a single or multi layers of high permeability metal. In [10], multi-layered core structures are proven to have better performance than single layered structures. By using the theoretical analysis given in [10], the root mean square (RMS) voltage induced at coil terminals for multi-layered core structures can be expressed as

$$
V_{\text {coil }}=\left(N_{C W E H}\right) \mu_{0} \mu_{r} \omega f I \ln \left(1+\frac{h}{r}\right),
$$

where $N_{C W E H}$ and $r$ denote the number of turns in the coil and the distance between coil and the conductor. $\mu_{0}=4 \pi 10^{-7}$ $\mathrm{H} / \mathrm{m}$ is the permeability of free space and $\mu_{r}$ is the relative permeability. $\omega$ and $h$ are width and the thickness of the core material. Finally, $I$ and $f$ represent the RMS magnitude and 
the frequency of the current. Since $\mu_{r}$ ignores the effect of the core gap, we modify $\mu_{r}$ to include core gaps. In the following equation, $\mu_{r g}$ shows the effective permeability accounting for the core gap which is calculated as

$$
\mu_{r g}=\frac{\frac{l_{m}}{A}}{\frac{1}{\frac{A}{l_{g}}+\left(0.241+\frac{1}{\pi} \ln \frac{b}{l_{g}}\right) p}+\frac{\frac{l_{m}}{A}}{\mu_{r}}} .
$$

In this equation $A=h \times \omega, p=2(h+\omega), b, l_{g}$, and $l_{m}$ are cross-sectional area of the core, perimeter of the cross section of the core, half the inner toroidal core perimeter, core gap length, and average core perimeter without the gap, respectively. $l_{m}$ can be calculated by using

$$
l_{m}=l_{a}-l_{g}=\frac{\pi\left(D_{o}-D_{i}\right)}{\ln \left(\frac{D_{o}}{D_{i}}\right)}-l_{g},
$$

where $l_{a}, D_{o}$, and $D_{i}=2 r=2 b / \pi$ parameters are average core perimeter, outer, and inner core diameters, respectively. If the magnetic core is considered as $n_{p}$-layered, then $\mu_{r g}$ can be improved as

$$
\mu_{r g n}=1+\left(\mu_{r g}-1\right) \frac{n_{p} \times d_{1}}{T} .
$$

The parameters, $n_{p}, d_{1}=h / n_{p}$, and $T$ are number of core layers, thickness of single core layer, and thickness of entire core, respectively. Maximum power delivered to $R_{\text {coil }}$ load resistance can be defined as [13]

$$
P_{C W E H}=\frac{\left(V_{\text {coil }} / 2\right)^{2}}{R_{\text {coil }}} \text {. }
$$

The load resistance, $R_{\text {coil }}$, is calculated as [13]

$$
R_{\text {coil }}=2 \pi\left(0.5 D_{o}\right)\left(N_{C W E H}\right) \rho+\frac{\pi N_{C W E H}^{2} d_{w i r e}^{2} \rho}{L_{c}},
$$

where $\rho, d_{\text {wire }}$, and $L_{c}$ are resistivity, diameter of the winding wire, and length of the core, respectively. For CWEH, we adopt the parameters given in [10], [13] which are listed as: $N_{C W E H}=280, \omega=50 \mathrm{~mm}, f=60 \mathrm{~Hz}, I=13.5 \mathrm{~A}, l_{g}=$ $1 \mathrm{~mm}, \mu_{r}=10^{5} \mathrm{H} / \mathrm{m}, d_{\text {wire }}=0.14 \mathrm{~mm}, d_{1}=0.1016 \mathrm{~mm}$, $T=0.78 \mathrm{~mm}, D_{o}=16.3 \mathrm{~mm}, D_{i}=14.32 \mathrm{~mm}, l_{m}=47.3$ $\mathrm{mm}, n_{p}=5, L_{c}=50 \mathrm{~mm}$, and $\rho=1.11 \Omega / \mathrm{m}$.

3) Free Standing Electromagnetic Harvester (FSEH): Free standing electromagnetic harvesters (FSEHs) are used in cases when CWEHs cannot be applied directly due to the distance constraints between the conductor and the harvesting coil. Since FSEHs cannot be clamped on top of the conductor, they can be placed near power cables, busbars and transformers. Hence, the gap assumption stated in Equation 6 is not valid [12]. According to the theoretical analysis provided in [12], the RMS voltage becomes

$$
V_{\text {coil }}=2 \pi f \mu_{e f f}\left(N_{F S E H}\right) \pi(0.5 D)^{2} B,
$$

where $N_{F S E H}$ is the number of turns in the coil, $B$ is the magnitude of the magnetic flux density parallel to the coil axis, $D$ is the diameter of the cylindrical core, and $1<\mu_{e f f}<\mu_{r}$ is the effective relative permeability. The maximum power can be harvested by using FSEHs (i.e., $P_{F S E H}$ ) is calculated by using Equation 9. For FSEH we used the parameters in [11], [12] as $N_{F S E H}=10 \mathrm{~K}, D=D_{o}=0.05 \mathrm{~m}, \mu_{e f f}=23 \mathrm{H} / \mathrm{m}$, and $B=10 \mu T_{r m s}$, respectively.

In Figure 2 we present $P_{C W E H}$ and $P_{F S E H}$ harvested powers with respect to $I$ (in Figure 2a) and $B$ (in Figure 2b) as functions of $n_{p}$ (in Figure 2a) and $N_{F S E H}$ (in Figure 2b) considering the parameters given above. Incrementing the number of layers $\left(n_{p}\right)$ in the magnetic core and number of turns in coils $\left(N_{F S E H}\right)$ increases the power harvested. Moreover, increasing the RMS magnitude of the current $(I)$ and flux density $(B)$ also increases the amount of harvested power.

4) Hybrid Energy Harvesting Model: In our hybrid energy harvesting model, we assume that each critical power substation has a sensor node for monitoring. In addition, substations are covered with SEH panels as well as CWEH and FSEH devices. During an hour interval, the weather is cloudy with probability $P_{\text {cloud }}$ independent of other 1-hour time intervals. If the weather is not cloudy during the day time (i.e., 5:00 a.m. to 7:00 p.m.), all sensor nodes utilize harvested solar energy according to the profile given in Figure 1. Otherwise, if the weather is cloudy or during the night time, CWEH or FSEH are used to harvest energy with equal probability. Harvested powers are converted to electrical energy and transferred to the battery of each node.

\section{MIP Framework for Energy Minimization}

In this section, we present the MIP framework that aims to minimize the energy dissipation of the highest energy consuming node (i.e., a MinMax problem), since the lifetime of WSNs is usually limited by the lifetime of the the highest energy consuming node, by considering the hybrid energy harvesting strategy as well as transmission power control. The network topology is considered as a directed graph $G=(V, \mathcal{A})$ with $V$ represents all nodes including the base station (node-1). We also define set $W$ which includes all nodes except node-1 (i.e., $W=V \backslash\{1\}) . \mathcal{A}=\{(i, j): i \in W, j \in V-i\}$ is the ordered set of arcs. Note that the set $\mathcal{A}$ implies that no node sends data to itself and the base station cannot generate data. The number of data packets flowing from node- $i$ to node- $j$ at time interval$t$ is represented with the integer variable $f_{i j}^{t}$. We define the set $\mathcal{T}=\{1,2, \ldots, 24\}$ to represent hours which is used to divide a single day to 24 hours (e.g., $t=1$ represents 12:00 a.m. to 1:00 a.m., $t=2$ represents 1:00 a.m. to 2:00 a.m. and so on). We represent each hour as 720 rounds (i.e., $N=720$ ) where a round duration is taken as 5 seconds (i.e., $\tau_{r n d}=5$ seconds). The MIP model is given in Figure 3. The objective function of the MIP model minimizes the highest energy dissipating node (i.e., E). Constraints of the MIP model are given in 12-20.

Constraint 12 ensures that incoming flows (i.e., $\sum_{j \in V} f_{i j}^{t}$ ), generated flows at each round (i.e., $N \times s_{i}$ ), and outgoing flows (i.e., $\sum_{j \in W} f_{j i}^{t}$ ) are balanced at each sensor node (i.e., $\forall i \in W$ ) in each time interval (i.e., $\forall t \in \mathcal{T}$ ). Constraint 13 enforces all sensor nodes to convey their collected data to the base station at each time interval-t. Constraint 14 is defined 


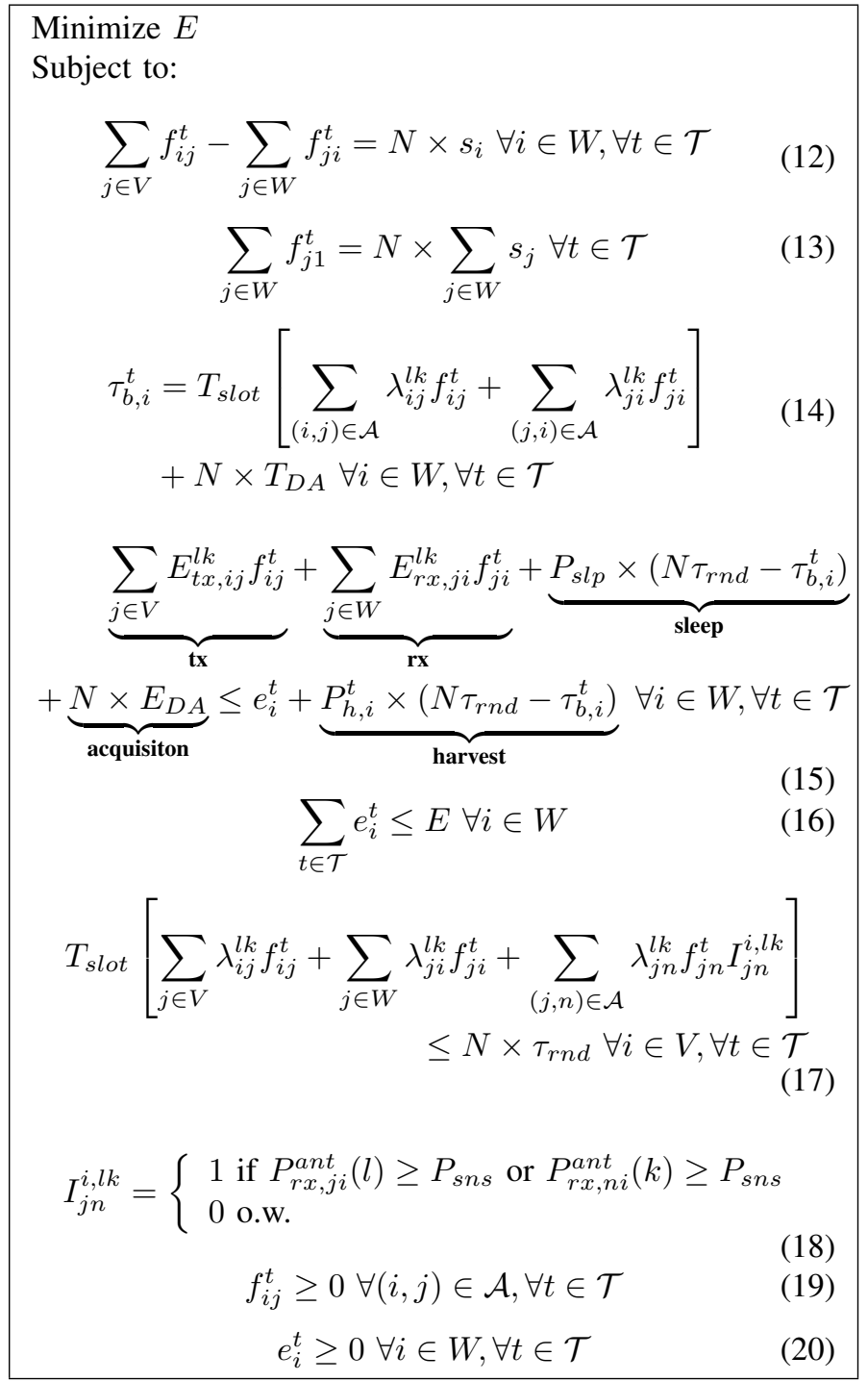

Fig. 3: MIP framework.

to calculate the busy time of node- $i$ (i.e., $\tau_{b, i}^{t}$ ) including the effects of retransmissions for each time interval- $t$. Note that $T_{D A}=5 \mathrm{~ms}$ is the time to acquire a data packet [14]. Lefthand side of Constraint 15 is used to calculate the total amount energy consumed for transmission, reception, sleep, and data acquisition at each node- $i$ for each time interval-t. $E_{D A}=57$ $\mu \mathrm{J}$ is the energy consumed for data acquisition and $P_{s l p}=3$ $\mu \mathrm{W}$ is the sleep power [14]. Right-hand side of this constraint bounds the total amount of energy dissipation to $e_{i}^{t}$ plus extra energy provided by the hybrid energy harvesting method. In this definition, $e_{i}^{t}$ variable denotes the amount of energy consumed by node- $i$ at time interval- $t$. We assume that energy harvesting is performed when node- $i$ is in the sleep mode which lasts $N \tau_{r n d}-\tau_{b, i}^{t}$ seconds at each time interval- $t$. In this constraint $P_{h, i}^{t}$ denotes the power harvested at node- $i$ in time interval- $t$ which is derived in the previous section. Constraint 16 is used to minimize the maximum energy consuming node in a single day (i.e., $\sum_{t \in \mathcal{T}} e_{i}^{t}$ ). Constraint 17 is used for each

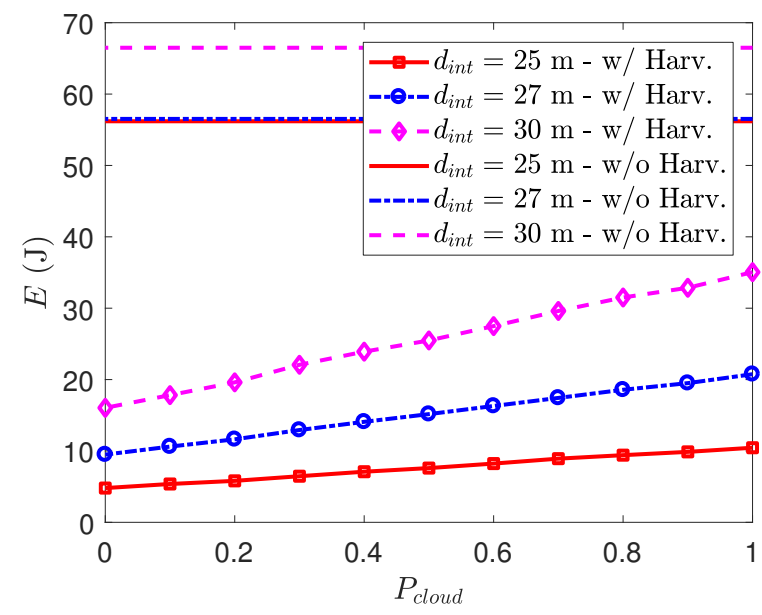

(a) Outdoor $500 \mathrm{kV}$ Substation (LOS)

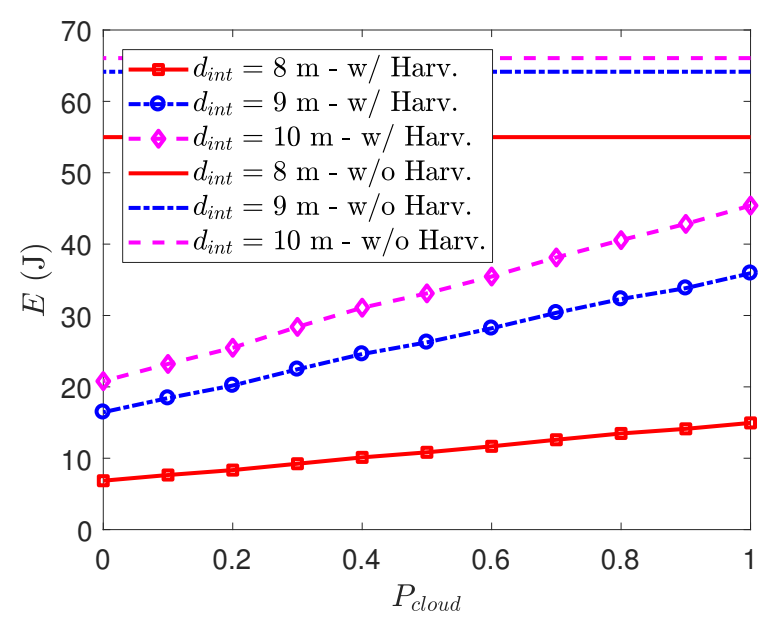

(b) Outdoor $500 \mathrm{kV}$ Substation (NLOS)

Fig. 4: Energy dissipation of the most energy consuming node ( $E$ in Joules) as a function of $P_{\text {cloud }}$ for the outdoor $500 \mathrm{kV}$ substation LOS and NLOS environments w/ and w/o energy harvesting on a grid topology considering several $d_{\text {int }}$ values.

node- $i$ to limit the aggregated duration of incoming, outgoing, and interfering flows to the total round duration of one hour (i.e., $N \times \tau_{r n d}$ ). Interference mechanism is stated in 18 and works as follows: if node- $i$ interferes the two-way data/ACK communications between node- $j$ and node- $n$ by using power levels- $l$ and $k$ as long as the reception power is greater than the nominal receiver sensitivity level (i.e., $P_{\text {sns }}=-94 \mathrm{dBm}$ ), the interference function for node- $i$ will be 1 . Finally, Constraints 19 and 20 show the non-negativity conditions of the variables. We employ a link-level transmission power control scheme to determine the optimal power level pair for data packet and ACK packet (i.e., $\left.\left\{l_{i j}^{o}, k_{j i}^{o}\right\}\right)$ transmissions that minimizes the total energy dissipation in each link (i.e., $E_{t x, i j}^{l k}+E_{r x, j i}^{l k}$ ).

\section{ANALYSIS}

In this section, we present the performance of our hybrid energy harvesting model on the energy consumption of sensor 
nodes. We construct the wireless channel model (Section II-A), link-layer model (Section II-B), and hybrid energy harvesting model (Section II-C) in MATLAB. The MIP model (Section II-D) is built and solved in GAMS by using CPLEX solver.

We consider an outdoor $500 \mathrm{kV}$ substation power grid environment where $|V|=49$ nodes are placed near to substations. Sensor nodes are placed to form a square (grid) topology where the base station is located at the center of the power grid. We note the distance between any node pairs in the same line as $d_{\text {int }}$. As in [1], we consider both LOS and NLOS cases while adopting $n$ and $\sigma$ values as 2.42 and $3.12 \mathrm{~dB}$ for LOS case; 3.51 and $2.95 \mathrm{~dB}$ for NLOS case. We choose $d_{\text {int }}$ values as 25, 27, 30 meters for LOS case; 8, 9, 10 meters for NLOS case to account for harsh SG environments. On each substation, SEH, CWEH, and FSEH devices are installed and harvested energy, according to the principle stated in Section II-C4, is transferred to the batteries of WSN nodes.

In Figure 4, we show our results by using the MIP model. We present the energy dissipation of the highest energy consuming node $(E)$ with respect to the probability of cloudy weather $\left(P_{\text {cloud }}\right)$ for LOS (in Figure $4 \mathrm{a}$ ) and NLOS cases (in Figure $4 \mathrm{~b}$ ) considering the aforementioned $d_{i n t}$ values. We also present the results for non-harvesting cases (i.e., w/o harvesting) in this figure as dashed lines by substituting $P_{h, i}^{t}$ with zero. Our results reveal that $E$ does not change with the $P_{\text {cloud }}$ when considering w/o harvesting. We observe that highest energy consumption of a node can be in the interval 56.19-66.49 Joules and 54.99-66.06 Joules for LOS and NLOS cases, respectively. We also note that $E$ increases as $d_{\text {int }}$ increases due to the raise of the transmission energy (i.e., $E_{t x, i j}^{l k}$ ) for a sparse network. The proposed hybrid energy harvesting model (i.e., w/ harvesting) can reduce $E$ by $47.29 \%$ to $91.46 \%$ and $31.27 \%$ to $87.54 \%$ for LOS and NLOS cases, respectively. It is quite revealing that amount of energy harvested is greatly increased (hence reducing $E$ ) as $P_{\text {cloud }}$ decreases. When solar energy is fully exploited (i.e., $P_{\text {cloud }}=0$ ), $75.88 \%-91.46 \%$ and $68.51 \%-87.54 \%$ of energy savings can be obtained for LOS and NLOS cases, respectively. On the other hand, when $P_{\text {cloud }}=1$, only CWEH and FSEH are utilized which would yield $47.29 \%-81.40 \%$ and $31.27 \%-72.81 \%$ of energy savings for LOS and NLOS cases, respectively.

\section{CONCLUSION}

In this work, a hybrid energy harvesting model that exploits both solar and electromagnetic energies is proposed for WSN based SG applications. The proposed hybrid harvesting method considers the stochastic nature of weather conditions into the account. An MIP framework is designed on top of a log-normal shadowing channel as the physical layer and handshaking based link layer model that can employ transmission power control on each link. The MIP framework is used as a benchmark for the analysis and minimizes the energy dissipation of the highest energy consuming WSN node. Quantitative analysis is performed on a square WSN topology deployed for an outdoor $500 \mathrm{kV}$ substation system for two different channel cases (LOS and NLOS). Our results reveal that developed hybrid energy harvesting framework can reduce the maximum energy consuming node up to $91.46 \%$ when solar energy is fully benefited. Nevertheless, when the solar energy cannot be exploited, the proposed hybrid harvesting method can still decrease the energy consumption up to $81.40 \%$.

\section{ACKNOWLEDGEMENT}

The work of V. C. Gungor was supported by the Turkish National Academy of Sciences Distinguished Young Scientist Award Program (TUBA-GEBIP) under Grand no. V.G./TUBAGEBIP/2013-14.

\section{REFERENCES}

[1] V. C. Gungor, B. Lu, and G. P. Hancke, "Opportunities and challenges of wireless sensor networks in smart grid," IEEE Transactions on Industrial Electronics, vol. 57, no. 10, pp. 3557-3564, 2010.

[2] D. Sahin, V. C. Gungor, T. Kocak, and G. Tuna, "Quality-of-service differentiation in single-path and multi-path routing for wireless sensor network-based smart grid applications," Ad Hoc Networks, vol. 22, pp. 43-60, 2014.

[3] B. E. Bilgin and V. C. Gungor, "On the performance of multi-channel wireless sensor networks in smart grid environments," in Proc. Int. Conference on Computer Communications and Networks (ICCCN), 2011, pp. 1-6.

[4] E. Fadel, V. C. Gungor, L. Nassef, N. Akkari, M. A. Malik, S. Almasri, and I. F. Akyildiz, "A survey on wireless sensor networks for smart grid," Computer Communications, vol. 71, pp. 22-33, 2015

[5] J. Han, J. Hu, Y. Yang, Z. Wang, S. X. Wang, and J. He, "A nonintrusive power supply design for self-powered sensor networks in the smart grid by scavenging energy from AC power line," IEEE Transactions on Industrial Electronics, vol. 62, no. 7, pp. 4398-4407, 2015.

[6] M. Erol-Kantarci and H. T. Mouftah, "Suresense: sustainable wireless rechargeable sensor networks for the smart grid," IEEE Wireless Communications, vol. 19, no. 3, pp. 30-36, 2012.

[7] R. Moghe, Y. Yang, F. Lambert, and D. Divan, "A scoping study of electric and magnetic field energy harvesting for wireless sensor networks in power system applications," in Proc. IEEE Energy Conversion Congress and Exposition, 2009, pp. 3550-3557.

[8] J. Hsu, S. Zahedi, A. Kansal, M. Srivastava, and V. Raghunathan, "Adaptive duty cycling for energy harvesting systems," in Proc. Int. Symposium on Low Power Electronics and Design (ISLPED), 2006, pp. $180-185$.

[9] R. Yao, W. Wang, M. Farrokh-Baroughi, H. Wang, and Y. Qian, "Quality-driven energy-neutralized power and relay selection for smart grid wireless multimedia sensor based IoTs," IEEE Sensors Journal, vol. 13, no. 10, pp. 3637-3644, 2013.

[10] R. H. Bhuiyan, R. A. Dougal, and M. Ali, "A miniature energy harvesting device for wireless sensors in electric power system," IEEE Sensors Journal, vol. 10, no. 7, pp. 1249-1258, 2010.

[11] K. Tanaka, Y. Mizuno, and K. Naito, "Measurement of power frequency electric and magnetic fields near power facilities in several countries," IEEE Transactions on Power Delivery, vol. 26, no. 3, pp. 1508-1513, 2011.

[12] N. M. Roscoe and M. D. Judd, "Harvesting energy from magnetic fields to power condition monitoring sensors," IEEE Sensors Journal, vol. 13, no. 6 , pp. 2263-2270, 2013.

[13] S. Yuan, Y. Huang, J. Zhou, Q. Xu, C. Song, and P. Thompson, "Magnetic field energy harvesting under overhead power lines," IEEE Transactions on Power Electronics, vol. 30, no. 11, pp. 6191-6202, 2015.

[14] S. Kurt, H. U. Yildiz, M. Yigit, B. Tavli, and V. C. Gungor, "Packet size optimization in wireless sensor networks for smart grid applications," IEEE Transactions on Industrial Electronics, vol. 64, no. 3, pp. 2392 2401, 2017.

[15] Tmote Sky Datasheet. [Online]. Available: http://www.eecs.harvard.edu/ konrad/projects/shimmer/references/tmotesky-datasheet.pdf 